\title{
Hausärztliche Highlights vom Kongress für Schlafmedizin
}

\section{WISSENSCHAFTLICH BESTÄTIGT}

\section{Wickelkinder schlafen besser}

In vielen Kulturen ist es üblich, Säuglinge zum Schlafen fest einzuwickeln (sogenanntes „swaddling“) und sie damit in ihrer Bewegungsfreiheit einzuschränken. In einer Studie wurde jetzt überprüft, ob an der Behauptung, dass die Kinder dann besser schlafen, etwas dran ist.

Dr. L. E. Meyer und seine Kollegen von der Universitätsklinik Cottbus ließen jeweils 30 gesunde Säuglinge im Alter von etwa sieben Wochen entweder fest gewickelt in ein „Wickelkissen“ oder in einem han- delsüblichen Schlafsack im Schlaflabor schlafen.

Das Ergebnis: Die fest eingewickelten Kinder hatten insgesamt einen ruhigeren Schlaf, wachten signifkant seltener spontan auf und zeigten eine höhere Schlafeffizienz. Somit scheint das auch von unseren Großmüttern noch praktizierte Einwickeln von Säuglingen tatsächlich den Schlaf zu verbessern. Eine gezielte Anwendung bei unruhigem Säuglingsschlaf ist einen Versuch wert, so die Autoren.

\section{RISIKOFAKTOR DICKER HALS}

\section{Kraftsport fördert Schlafapnoe}

Als einer der Hauptrisikofaktoren für schlafbezogene Atemstörungen gilt ein vergrößerter Halsumfang von mehr als $45 \mathrm{~cm}$ bei Männern und mehr als $36 \mathrm{~cm}$ bei Frauen. Solch einen dicken Hals haben aber nicht nur Fettleibige, sondern auch Bodybuilder und Kraftsportler mit hypertrophierter Muskulatur.

C. Brocks untersuchte polygrafisch Probanden, die seit mindestens drei Jahren intensives Bodybuilding betrieben. Ihnen gegenüber gestellt wurden normalgewichtige Männer, deren Fitnesstraining nicht über ein gesundheitsorientiertes Trainingsprogramm hinausging. Wie die Un-

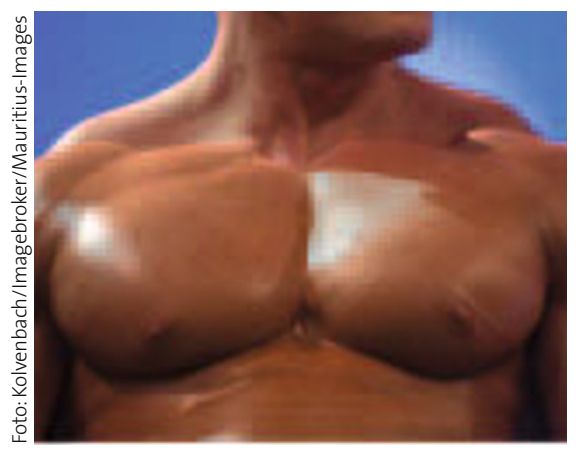

Solche Muskelpakete können im Wortsinn atemberaubend sein.

tersuchung zeigte, hatten die Bodybuilder einen signifikant höheren ResipiratoryDistress-Index und ein signifkant erhöhtes Risiko für eine obstruktive Schlafapnoe.

\section{UNGÜNSTIGE WECHSELWIRKUNG VERMUTET}

\section{Gestörter Schlaf: Risikofaktor für chronischen Schmerz?}

Schlafstörungen gehören zu den am häufigsten genannten Beschwerden von Patienten mit chronischen Schmerzsyndromen. Neuere Untersuchungen legen nahe, dass umgekehrt auch ein gestörter Schlaf zur Entstehung der chronischen Schmerzen beitragen kann.

Wie Dr. M. Smith aus Baltimore, USA, berichtete, zeigen mehrere experimentelle Studien, dass Schlafmangel die Schmerzsensitivität direkt beeinflussen kann. Damit ist es auch denkbar, dass Insomnie und Schlafmangel einen Risikofaktor für die Exazerbation und Chronifizierung von Schmerzen darstellen könnte. Erste Daten weisen in diese Richtung. So wurde gezeigt, dass bei Patienten mit schweren Brandverletzungen das Vorhandensein von Schlafstörungen ein unabhängiger Prädiktor der Entstehung von chronischen Schmerzsyndromen ist. Störungen der Schlafkontinuität scheinen die endogene Schmerzhemmung negativ zu beeinflussen. Dies könnte auch bei idiopathischen Schmerzsyndromen wie der Fibromyalgie von Bedeutung sein.

\section{BEI SCHLAFSTÖRUNGEN}

\section{Viel Bewegung für die nötige Bettschwere?}

Dass regelmäßige körperliche Bewegung einen gesunden Schlaf fördert, gilt als Binsenweisheit. Eine Untersuchung aus der Schweiz stellt diesen Zusammenhang aber zumindest bei jungen Erwachsenen infrage.

Dr. S. Brand und Kollegen aus Basel untersuchten Schlaf, körperliche Bewegung und psychisches Wohlbefinden von 837 Studentinnen und Studenten. Dabei stellte sich heraus, dass die Menge an körperlicher Aktivität weder mit der Schlafdauer noch mit der Schlafqualität korreliert war. Einen eindeutig negativen Einfluss auf die Schlafqualität hatten dagegen depressive und hypomanische Symptome. Auch auf das Ausmaß der körperlichen Aktivität wirkten sich Stimmungstiefs negativ aus. Beides war aber unabhängig voneinander.

Der einfache Rat an Patienten mit Schlafstörungen, mit viel Bewegung für die nötige Bettschwere zu sorgen, greift somit möglicherweise zu kurz.

SCHLAFBEZOGENE ATMUNGSSTÖRUNGEN

\section{Bei KHK-Patienten lohnt ein Screening}

Jeder zweite KHK-Patienten leidet unter einer abklärungs- und wahrscheinlich auch therapiebedürftigen schlafbezogenen Atemstörung (SAS). Das zeigt eine Screeninguntersuchung bei den KHK-Patienten einer Klinik in Bad Rothenfelde. Innerhalb eines Jahres wurde bei allen 496 KHK-Patienten routinemäßig eine Polygrafie durchgeführt. Völlig frei von SAS waren nur $13,7 \%$, berichtete Dr. H.-C. Purucker. Bei Männern waren die Störungen deutlich ausgeprägter als bei Frauen. Die Autoren empfehlen, das SASScreening zumindest in kardiologischen Rehabilitationseinrichtungen zur Routine werden zu lassen.

MARIA WEIß .

- Jahrestagung der Deutschen Gesellschaft für Schlafmedizin, Kassel, 16.-18. Oktober 2008 